\title{
Multiple $\mathrm{m}^{6} \mathrm{~A}$ RNA methylation modulators promote the malignant progression of hepatocellular carcinoma and affect its clinical prognosis
}

Nanfang Qu ${ }^{1,2}$, Sanyu Qin ${ }^{1}$, Xuemei Zhang ${ }^{3}$, Xiaotong Bo ${ }^{2}$, Zhengchun Liư ${ }^{4}$ Chao Tan ${ }^{5}$, Guiqiong Wen ${ }^{6}$ and Haixing Jiang ${ }^{1 *}$

\begin{abstract}
Background: Hepatocellular carcinoma (HCC) is the second most common cause of cancer-related death in the world. $N^{6}$-methyladenosine $\left(m^{6} \mathrm{~A}\right)$ RNA methylation is dynamically regulated by $\mathrm{m}^{6} \mathrm{~A}$ RNA methylation modulators ("writer," "eraser," and "reader" proteins), which are associated with cancer occurrence and development. The purpose of this study was to explore the relationships between $\mathrm{m}^{6} \mathrm{~A}$ RNA methylation modulators and HCC.

Methods: First, using data from The Cancer Genome Atlas (TCGA) and International Cancer Genome Consortium (ICGC) databases, we compared the expression levels of 13 major m6A RNA methylation modulators between HCC and normal tissues. Second, we applied consensus clustering to the expression data on the $\mathrm{m}^{6} \mathrm{~A}$ RNA methylation modulators to divide the HCC tissues into two subgroups (clusters 1 and 2), and we compared the clusters in terms of overall survival (OS), World Health Organization (WHO) stage, and pathological grade. Third, using least absolute shrinkage and selection operator (LASSO) regression, we constructed a risk signature involving the $\mathrm{m}^{6} \mathrm{~A}$ RNA methylation modulators that affected OS in TCGA and ICGC analyses.

Results: We found that the expression levels of 12 major m6A RNA methylation modulators were significantly different between HCC and normal tissues. After dividing the HCC tissues into clusters 1 and 2, we found that cluster 2 had poorer OS, higher WHO stage, and higher pathological grade. Four $\mathrm{m}^{6} \mathrm{~A}$ RNA methylation modulators (YTHDF1, YTHDF2, METTL3, and KIAA1429) affecting OS in the TCGA and ICGC analyses were selected to construct a risk signature, which was significantly associated with WHO stage and was also an independent prognostic marker of OS.
\end{abstract}

Conclusions: In summary, $\mathrm{m}^{6} \mathrm{~A}$ RNA methylation modulators are key participants in the malignant progression of HCC and have potential value in prognostication and treatment decisions.

Keywords: m6A, RNA methylation, Liver hepatocellular carcinoma, Prognosis

\footnotetext{
*Correspondence: bosjianghx@163.com

${ }^{1}$ Department of Gastroenterology, The First Affiliated Hospital of Guangxi

Medical University, Nanning 530021, China

Full list of author information is available at the end of the article
}

(c) The Author(s). 2020 Open Access This article is distributed under the terms of the Creative Commons Attribution 4.0 International License (http://creativecommons.org/licenses/by/4.0/), which permits unrestricted use, distribution, and reproduction in any medium, provided you give appropriate credit to the original author(s) and the source, provide a link to the Creative Commons license, and indicate if changes were made. The Creative Commons Public Domain Dedication waiver (http://creativecommons.org/publicdomain/zero/1.0/) applies to the data made available in this article, unless otherwise stated. 


\section{Background}

According to the Cancer Statistics of China in 2015, hepatocellular carcinoma ( $\mathrm{HCC})$ is the second and fifth most common cause of cancer-related death in men and women, respectively [1].Worldwide, $\mathrm{HCC}$ is the most common type of primary liver cancer, ranking second among malignant tumors, with more than 700,000 deaths each year [2]. Chronic liver inflammation caused by hepatitis $\mathrm{B}$ virus (HBV), hepatitis $\mathrm{C}$ virus (HCV), or aflatoxin $\mathrm{B} 1$ is the main cause of HCC [3]. A series of treatments have been developed for patients with HCC, including surgery, chemotherapy, and radiotherapy. However, the mortality rate is still very high [4-6]. Understanding the molecular mechanisms underlying the development of $\mathrm{HCC}$ is essential to improve diagnostic tools and treatments.

RNA methylation in mammalian cells is dynamic and reversible and has been suggested as another kind of epigenetic regulation analogous to DNA methylation or histone modification [1, 7]. $\mathrm{N}^{6}$-methyladenosine $\left(\mathrm{m}^{6} \mathrm{~A}\right)$ is considered the most frequent, abundant, and conserved internal modification of eukaryotic messenger RNA (mRNA) [8], microRNAs (miRNAs) [9], and long non-coding RNA [10]. The $\mathrm{m}^{6} \mathrm{~A}$ modification occurs at a consistent motif, $\mathrm{RRm}^{6} \mathrm{ACH}\left([\mathrm{G} / \mathrm{A} / \mathrm{U}][\mathrm{G}>\mathrm{A}] \mathrm{m}^{6} \mathrm{AC}[\mathrm{U}>\mathrm{A}>\mathrm{C}]\right)$ [11]. In addition, experiments using $\mathrm{m}^{6} \mathrm{~A}$-specific antibodies and high-throughput sequencing (HTSeq) revealed that $\mathrm{m}^{6} \mathrm{~A}$ modifications mainly appear around termination codons, in 3 '-untranslated regions, and in long internal exons [12]. These modifications play key roles in 3 '-end processing, pre-RNA splicing, translation regulation, nuclear output, miRNA processing, and RNA attenuation [13].

The $\mathrm{m}^{6} \mathrm{~A}$ modification can be reversed in a process coordinated by methyltransferases (m6A "writers"), demethylases $\left(\mathrm{m}^{6} \mathrm{~A}\right.$ "erasers"), and $\mathrm{m}^{6} \mathrm{~A}$ "reader" proteins [14]. The $\mathrm{m}^{6} \mathrm{~A}$ "writer" complex is composed of METTL3, WTAP, RBM15, METTL14, VIRMA (KIAA1429), and ZC3H13 [13, 15]. So far, only FTO and ALKBH5 have been identified as $\mathrm{m}^{6} \mathrm{~A}$ "erasers" [16]. As for $\mathrm{m}^{6} \mathrm{~A}$ "readers," including YTHDF1-2, YTHDC1-2, and HNRNPC, they can recognize $\mathrm{m}^{6} \mathrm{~A}$ modifications and consequently direct RNA alternative splicing, localization, translation, and stability, among other processes $[17,18]$. Thus, $\mathrm{m}^{6} \mathrm{~A}$ modifications direct mRNAs to different destinies through differential processing, translation, and decay during the stress response, embryonic development, and cell differentiation [19]. Increasing evidence has shown that genetic changes (e.g., copy number variation $[\mathrm{CNV}]$ and single-nucleotide polymorphism [SNP] mutations) and expression disorders related to $\mathrm{m}^{6} \mathrm{~A}$ RNA methylation modulators are closely related to the malignant progression of various cancers [20-24]. Many studies have pointed out that abnormal $\mathrm{m}^{6} \mathrm{~A}$ modification is associated with $\mathrm{HCC}$ progression [20, 25-29]. However, the role of $m^{6} \mathrm{~A}$ RNA methylation modulators in the malignant progression of $\mathrm{HCC}$ and their impact on prognosis has not been fully analyzed.
In this study, the expression in $\mathrm{HCC}$ tissues of 13 widely reported $\mathrm{m}^{6} \mathrm{~A}$ RNA methylation modulators was systematically analyzed using RNA sequencing data from The Cancer Genome Atlas (TCGA) and the International Cancer Genome Consortium (ICGC). Additionally, CNV and SNP mutations of these 13 modulators in the TCGA database were explored. According to the expression of $\mathrm{m}^{6} \mathrm{~A}$ RNA modulators, the HCC tissues were divided into clusters 1 and 2, and there were differences in the clinicopathological factors between the two groups. Furthermore, four $\mathrm{m}^{6} \mathrm{~A}$ RNA methylation modulators were selected to construct a least absolute shrinkage and selection operator (LASSO) risk regression model (a four-gene risk signature) to predict HCC prognosis in terms of survival.

\section{Methods \\ Datasets}

RNA sequencing transcriptome data and corresponding clinicopathological data were acquired for $374 \mathrm{HCC}$ and 50 normal tissues from TCGA (http://cancer.gov/) and 243 HCC and 202 normal tissues from ICGC (https:// dcc.icgc.org/releases/current/Projects/LIRI-JP). We used Liver Cancer, RIKEN, Japan [LIRI-JP] data, because the Chinese datasets are incomplete. For the TCGA transcriptome analysis, we downloaded HTSeq-fragments per kilobase of transcript per million mapped reads (FPKM) data. Clinicopathological data from the TCGA and ICGC datasets are presented in Table S1. We deleted the TCGA data on all samples with incomplete data regarding age, sex, World Health Organization (WHO) stage, and pathological grade in the analyses exploring the relationships between clinicopathological factors and HCC subtype (cluster 1 or 2) and between clinicopathological factors and the risk score; this led to $n=350$ HCC samples in these TCGA analyses. In the survival analysis, we deleted the data on samples with "futime" variable (survival or censoring time) $=0$; this led to $n=371$ HCC samples in the TCGA analysis.

Regarding the CNV and SNP analyses, we used TCGA data. Using the official TCGA website, we entered the Genomic Data Commons (GDC) Data Portal and selected "Liver" as the Primary Site and "TCGA-LIHC" as the Project. By downloading the Copy Number Variation in the Masked Copy Number Segment data type from the TCGA database, CNV data were obtained, comprising data on 379 HCC and 389 normal tissues. By downloading the Simple Nucleotide Variation of Masked Somatic Mutation data type from the TCGA database, SNP data were obtained, and then we analyzed these data that included 364 HCC tissues (TCGA.LIHC.varscan.40fe9c1b-19d0-45cf898a-f7b0cbad783e.DR-10.0.somatic.maf file). 


\section{Bioinformatics analysis}

We used ActivePerl 5.24.3 Build 2404 (https://www.perl. org), R v3.6.0 (https://www.r-project.org/), and SPSS v23.0 (IBM Corp., Armonk, NY, USA) to conduct data conversion, statistical analysis, and calculations. All our R and Perl packages were obtained from the Shengxin Selflearning Network (https://www.biowolf.cn/).

To compare the expression of 13 genes encoding $\mathrm{m}^{6} \mathrm{~A}$ RNA methylation modulators between HCC and normal tissues, we used the R package "pheatmap."

To investigate the function of $\mathrm{m}^{6} \mathrm{~A}$ RNA methylation modulators in $\mathrm{HCC}$, we clustered the $\mathrm{HCC}$ tissues in the TCGA dataset into two groups using the "ConsensusClusterPlus" and "limma" packages. We used the principal component analysis "PCA" package to explore the feasibility of tumor typing based on $\mathrm{m}^{6} \mathrm{~A}$-related gene expression.

To explore the prognostic value of $\mathrm{m}^{6} \mathrm{~A}$ RNA methylation modulators, univariate Cox regression analyses were performed using the expression levels of the 13 genes in the TCGA dataset. Thus, we identified nine genes that were significantly related to survival $(P<0.05)$, and further we used a LASSO regression to narrow the range of target genes because the predictor variable was much larger than the sample content in the gene expression data [30]. Finally, five genes were obtained.We took the same approach using ICGC data and obtained seven genes. We then selected the four genes that were common to both analyses for further analysis. We conducted another LASSO analysis of the four genes and selected the best penalty parameter $(\lambda)$. We obtained the same results using the two databases. Finally, the four genes were used to construct a risk signature. The risk score based on the signature was calculated using the following formula [31]:

$$
\text { Risk score }=\sum n_{i}=\sum\left(\text { Coefi } \times x_{i}\right)
$$

where Coef $_{i}$ is the coefficient and $x_{i}$ is the z-scoretransformed relative expression value of each selected gene. We used this formula to calculate a risk score for each patient in both the training (TCGA) and validation (ICGC) datasets.

To explore the prognostic value of the four-gene risk signature, first, we combined gene expression data with survival status and time data. HCC patients in the TCGA $(n=365)$ and ICGC $(n=232)$ datasets were divided into low- and high-risk groups based on the median risk score, and we compared the overall survival (OS) rate between the two groups using the Kaplan-Meier method.We also constructed nomograms based on the risk score and clinicopathological factors to predict 1-, 3-, and 5-year survival rates, using the R packages "Hmisc," "lattice," "Formula," "ggplot2," "foreign," and "rms," Finally, we verified the results sing the ICGC data.

\section{Statistical analysis}

To identify differential expression between HCC and normal tissues, the homogeneity of variance assumption was assessed and found not to be satisfied, so Wilcoxon rank-sum tests were used rather than $t$ tests. The chisquare test was used to compare $\mathrm{CNV}$ variation rates between the normal and tumor groups. For comparison of $\mathrm{CNV}$ among multiple groups (i.e., one copy lost, normal, one copy gained, and two or more copies gained), the homogeneity of variance assumption was assessed and found not to be satisfied, so Kruskal-Wallis tests were used rather than one-way analysis of variance.

After clustering the patients into two clusters based on consensus expression of the $\mathrm{m}^{6} \mathrm{~A}$ RNA methylation modulators, we used chi-square tests to compare the distribution of age, sex, WHO stage, and pathological grade between clusters 1 and 2 [31].

The Kaplan-Meier method and bilateral log-rank test were used to compare OS between HCC subtypes cluster 1 and 2 and between high- and low-risk groups (based on the median risk score calculated using the four-gene risk signature). The prediction efficiency of the risk score for 3-year survival was assessed using a receiver operating characteristic (ROC) curve analysis.

Univariate Cox regression analyses were used to assess the associations between the expression levels of the 13 genes and OS. Additionally, uni- and multivariate Cox regression analyses were used to determine the prognostic values of the risk score and various clinicopathological factors.

\section{Results}

\section{Most $\mathrm{m}^{6} \mathrm{~A}$ RNA methylation modulators are upregulated in $\mathrm{HCC}$}

TCGA transcriptome data showed that all 13 genes except for $\mathrm{ZC} 3 \mathrm{H} 13$ and METTL14 were differentially highly expressed in HCC tissues compared with normal tissues (Fig. 1A, Table S1). Further verification using ICGC data showed that all 13 genes except for METTL14 were differentially highly expressed in HCC tissues compared with normal tissues (Fig. 1B, Table S1).

\section{CNV and SNP analyses regarding $\mathrm{m}^{6} \mathrm{~A}$ RNA methylation modulators in HCC}

To understand whether the differential expression of the $\mathrm{m}^{6} \mathrm{~A}$ RNA methylation modulators is caused by genetic changes related to the corresponding genes, we analyzed the CNV and SNP data from the TCGA database. First, we found that the $\mathrm{CNV}$ of the 13 genes in HCC tissues was significantly different from that in normal tissues. More specifically, three genes (YTHDF1, YTHDC2, and KIAA1429) mainly exhibited increased copy numbers in HCC tissues, while the remaining 10 genes mainly exhibited decreased copy numbers (Figure S1A, Table S1). 

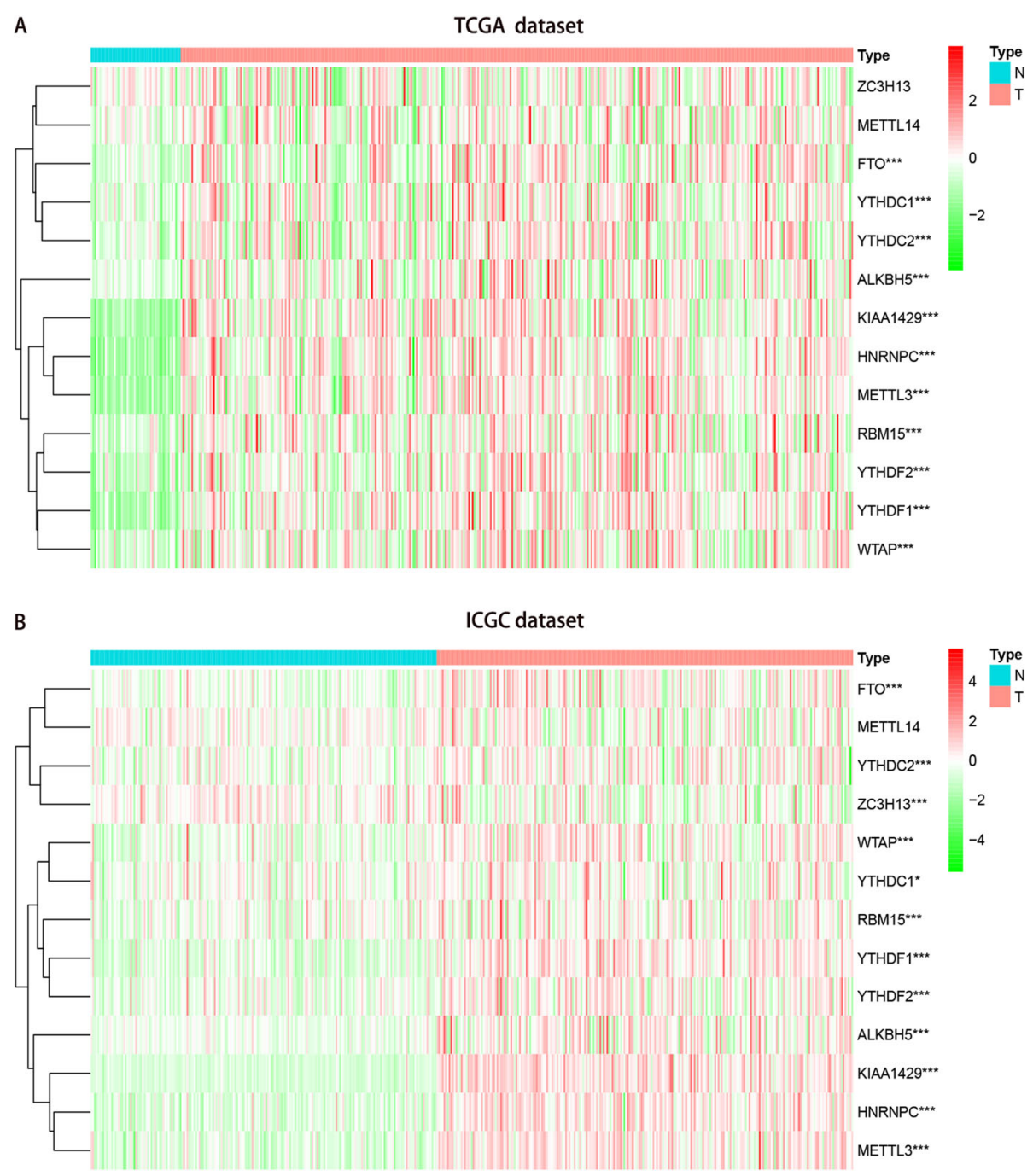

Fig. 1 Expression of m6A RNA methylation modulators in hepatocellular carcinoma. a Data from TCGA dataset; b Data from ICGC dataset; Wilcox test was used to determine the differential gene expression between tumor group and normal group. ${ }^{*} P<0.05$ and ${ }^{* * *} P<0.001$

Second, we explored the relationships between mRNA expression and copy number and found that all 12 genes upregulated in the TCGA analysis except for HNRNPC exhibited significantly increased copy numbers (Figure S2).

Furthermore, we observed that SNP mutations in the 13 genes were very low (all $\leq 1.9 \%$ ) in $\mathrm{HCC}$ tissues (Figure S1B). However, as mentioned earlier, the expression of most $\mathrm{m}^{6} \mathrm{~A}$ RNA methylation modulators was increased in HCC tissues compared to normal tissues, indicating that these changes in gene expression are not entirely caused by $\mathrm{CNV}$ or SNP mutations in the corresponding genes.

\section{Consensus clustering of m6A RNA methylation} modulators identified two clusters of HCC tissues which were associated with clinicopathological factors

Based on the similarity of expression levels of the m6A RNA methylation modulators, we observed the clustering stability of the TCGA dataset from $\mathrm{k}=2$ to 9 (Figures $\mathrm{S} 3 \mathrm{~A}-\mathrm{E})$. It can be seen that $\mathrm{k}=2$ seems to be an appropriate choice. Subsequently, we analyzed the differential gene expression of the two subgroups, designated as clusters 1 and 2 , and found that the expression of m6A RNA methylation modulators was higher in cluster 2 than in cluster 1 (Figure S3F). We then compared the clinicopathological factors between the two clusters. Cluster 1 was significantly associated with male sex $(P<0.05)$, lower pathological grade $(P<0.001)$, and lower WHO stage $(P<$ 0.05 ), while cluster 2 was significantly associated with female sex, higher pathological grade, and higher WHO stage (Fig. 2A). We further found that cluster 2 had significantly shorter OS than cluster 1 (Fig. 2B). Next, we used PCA to explore the feasibility of tumor typing based on m6A-related gene expression. The results showed that there were significant differences between 


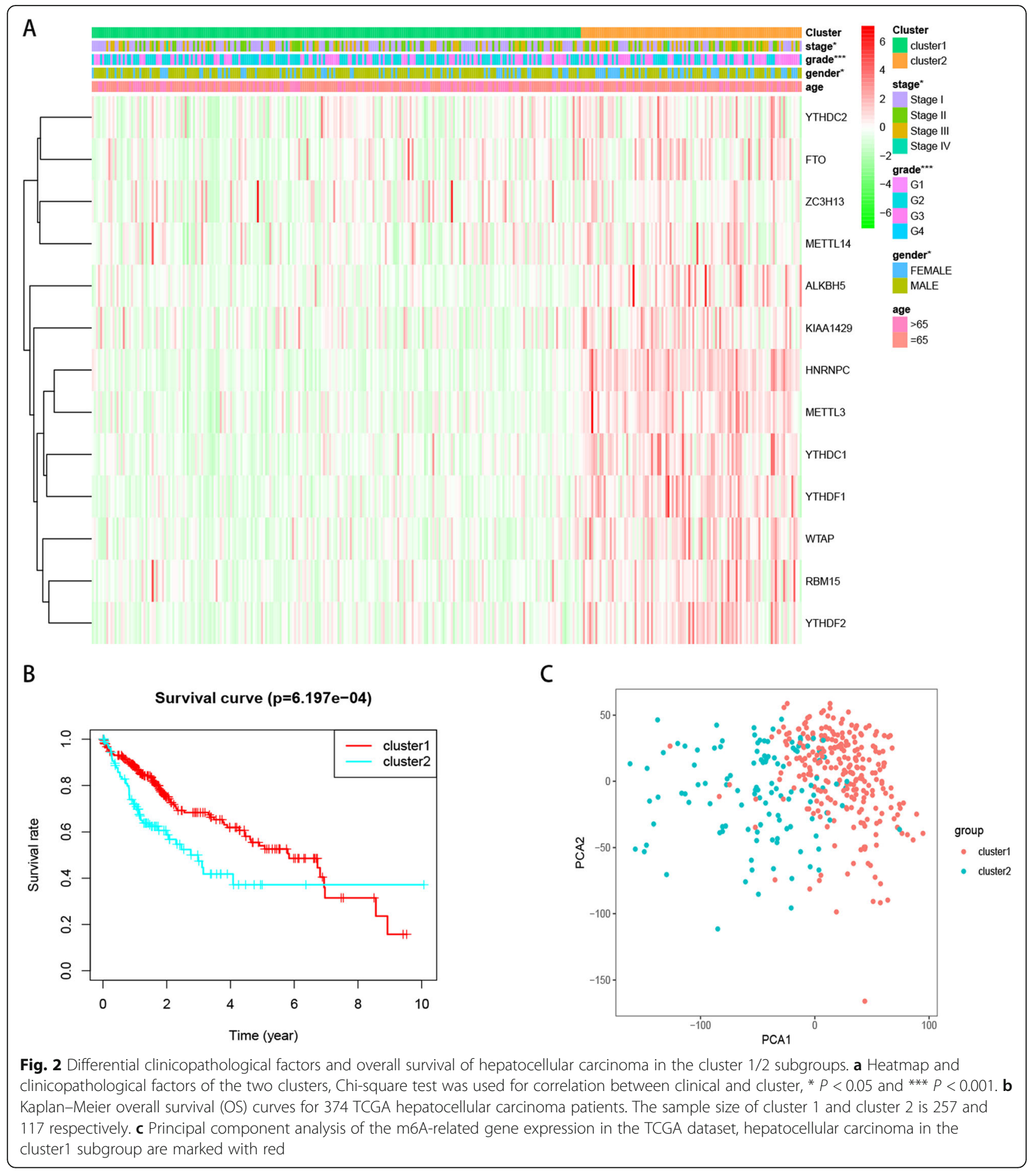

the two tumor subtypes and tumor typing was feasible (Fig. 2C).

\section{Prognostic value of $\mathrm{m}^{6} \mathrm{~A}$ RNA methylation modulators and risk signature}

Next, we explored the prognostic value of $\mathrm{m}^{6} \mathrm{~A}$ RNA methylation modulators in HCC. Univariate Cox regression analyses of TCGA data were performed to assess the associations between the expression levels of the 13 genes and OS (Fig. 3A). Nine genes were significantly associated with OS $(P<0.05)$. Among these nine genes, all but $\mathrm{ZC} 3 \mathrm{H} 13$ were high-risk genes with hazard ratios (HRs) $>1$, while ZC3H13 was a protective gene with $\mathrm{HR}<1$. We then used LASSO Cox regression to analyze the prognostic value of 
the nine genes in the TCGA dataset, and selected five genes based on the minimum criterion (Figs. 3B-D).

Additionally, univariate Cox regression analyses of ICGC data were performed to assess the associations between the expression levels of the 13 genes and OS (Fig. 3E). Seven genes were significantly associated with OS $(P<0.05)$. Among these seven genes, all but YTHDC2 were high-risk genes with HRs $>1$, while YTHDC2 was a protective gene with $\mathrm{HR}<1$. Additionally, we used LASSO Cox regression to analyze the prognostic value of the seven genes in the ICGC dataset, and selected seven genes based on the minimum criterion (Figs. 3F-H).

Thereafter, we selected the common genes (YTHDF1, YTHDF2, METTL3, and KIAA1429) in the two database analyses to construct a four-gene risk signature based on the minimum criterion. We used the coefficients obtained using LASSO Cox regression to calculate prognostic risk scores for cases in both the training TCGA dataset (Figure S4A) and the validation ICGC dataset (Figure S4B).

\section{Risk scores showed strong associations with survival and clinicopathological factors in HCC}

Patients with HCC in the TCGA datasets were divided into low- and high-risk groups based on the median risk score, and a significant difference in OS was found between the two groups (Figs. 4A-B). Additionally, ROC curve analysis showed that the risk score could predict the 3-year survival rate of HCC patients (Figs. 4C-D).

The heatmap of the expression levels of the four selected $\mathrm{m}^{6} \mathrm{~A}$ RNA methylation modulators in high- and low-risk patients in the TCGA dataset shows that the expression levels of the four genes were associated with increased risk scores (Figure S5A). Notably, survival rate and time were significantly reduced in the high-risk group compared to the low-risk group (Figure S5B). We constructed nomograms based on the risk score and clinicopathological factors to predict 1-, 3-, and 5-year survival rates (Figure S5C). Finally, we verified these trends using the ICGC data (Figures S6A-C).Next, using TCGA data, we conducted chi-square tests to determine whether we could better predict HCC clinical outcomes based on m6A RNA methylation modulators, and we found significant differences between low- and high-risk groups in WHO stage $(P<0.01)$, pathological grade $(P<$ $0.001)$, and cluster $1 / 2$ subgroups $(P<0.001$, Fig. $5 \mathrm{~A})$. Chi-square tests showed that four genes were highly expressed in the high-risk group and decreased in the low-risk group. We explored the relationship between the risk score and each clinicopathological factor with Wilcoxon rank-sum tests. In the TCGA dataset, the higher the risk score, the higher the WHO stage and the worse the pathological grade (Figs. 5B-D); the risk score was higher in cluster 2 than in cluster 1 .
Subsequently, univariate and multivariate Cox regression analyses of survival were performed using the risk score and relevant clinicopathological factors (age, sex, pathological grade, and WHO stage) in the TCGA dataset to determine whether the risk score (based on the fourgene risk signature) can be used as an independent prognostic factor. Both univariate and multivariate analyses showed that the risk score could be used as a prognostic indicator (both $P<0.001$, Figs. $5 \mathrm{E}-\mathrm{F}$ ). WHO stage was also an independent prognostic indicator and the $P$ value for age was near $0.05(0.073)$, but sex and pathological grade were not independent prognostic indicators.

Additionally, we verified the results in the ICGC database. Owing to the lack of data on pathological grade, the relationships between the risk score and clinicopathological factors could not be fully investigated. However, the relationship between risk score and $\mathrm{WHO}$ stage was consistent with the TCGA analysis $(P<0.01$, Fig. 6A). We also conducted univariate and multivariate regression analyses and, as in the TCGA analysis, the risk score and WHO stage were significantly associated with OS. Unlike in the TCGA analysis, there was a significant difference in sex in the ICGC analysis, with male sex leading to increased $\operatorname{OS}(P<0.05$ for both the univariate and multivariate analyses, Figs. 6B-C).

Furthermore, we constructed nomograms (involving risk score and clinicopathological factors) to predict 1-, 3-, and 5-year survival using TCGA (Figure S7A) and ICGC (Figure S7B) data. Regarding the TCGA nomograms, according to the above uni- and multivariate regression analyses using TCGA data, pathological grade was not an independent prognostic factor, so it was not included in the nomograms, while the $P$ value for age in the multivariate regression analysis was 0.073 (close to 0.05), so it was included (Figure S7A). In the ICGC nomograms, age was included to be consistent with the TCGA nomograms. Sex was also included because it was a prognostic factor in the above uni- and multivariate regression analyses using ICGC data (Figure S7B).

\section{Discussion}

HCC has been reported to be the second most common cause of cancer-related death worldwide [1]. HBV and $\mathrm{HCV}$ infections have resulted in a high rate of $\mathrm{HCC}$ in China. Because of the current lack of effective interventions, high metastasis rate, and high mortality rate, it is crucial to develop a deeper understanding of the molecular mechanism of HCC development. A growing body of evidence suggests that liver cancer is a multistep process associated with complex interactions between genetics, epigenetics, and transcriptional changes [32].

About 100 post-transcriptional chemical modifications can occur in biological RNAs [33] and $\mathrm{m}^{6} \mathrm{~A}$, which was discovered in the 1970s, is one of the most abundant 


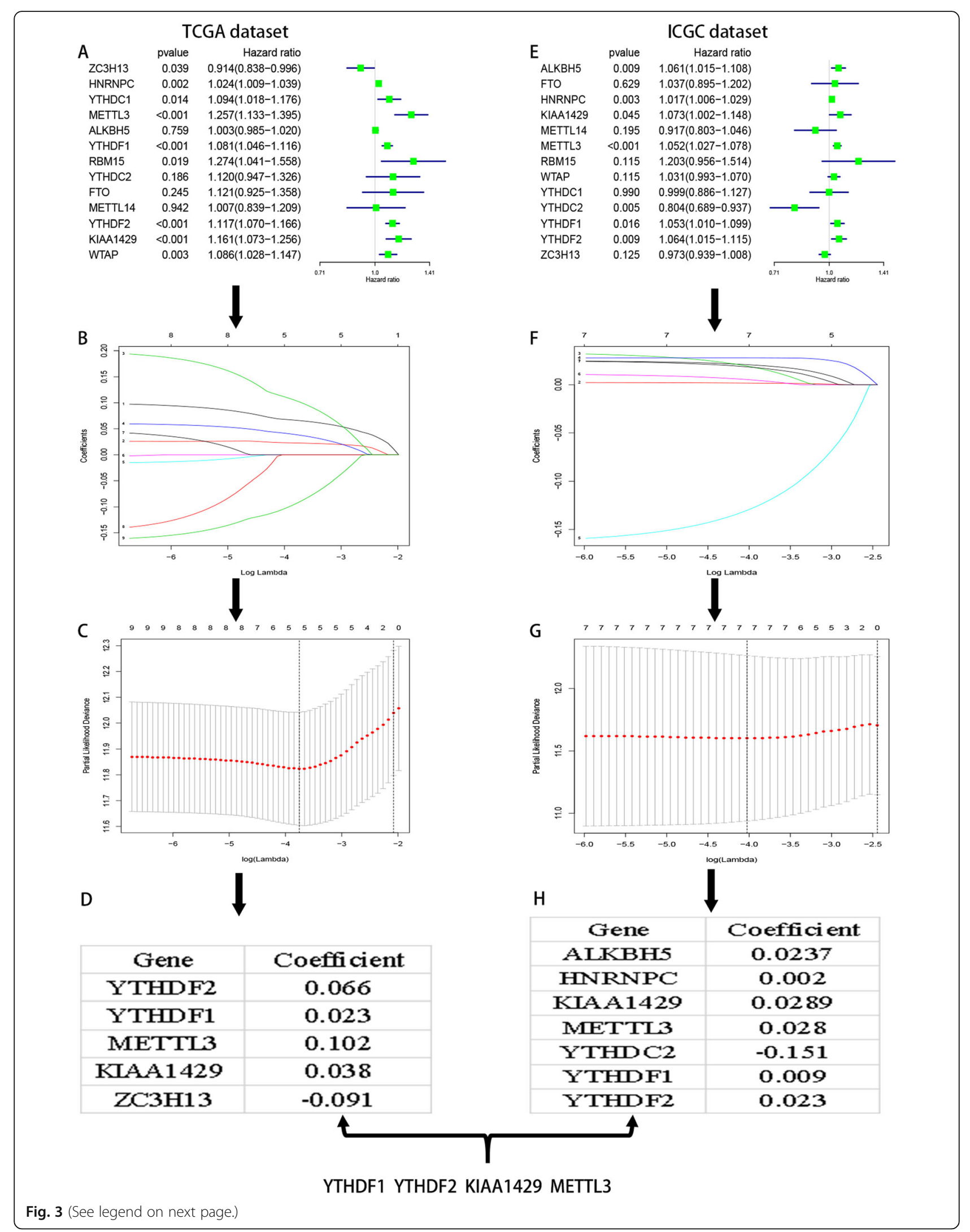


(See figure on previous page.)

Fig. 3 The process of selecting target genes to construct lasso risk regression model by applying the least absolute shrinkage and selection operator (LASSO) Cox regression algorithm. a Univariate Cox regression analysis of the 13 genes correlated with OS in TCGA dataset; the hazard ratios (HR), 95\% confidence intervals .c-d The process of selecting target genes in TCGA dataset. e-h The process of selecting target genes in ICGC dataset

endochemical modifications in eukaryotic mRNAs [8]. The biological significance of $\mathrm{m}^{6} \mathrm{~A}$ RNA methylation has been increasingly recognized; it has important and diverse biological functions in mammals, including sex determination, tissue development, DNA damage response, circadian rhythm, and tumorigenesis [34]. In this study, we demonstrated that the expression of $\mathrm{m}^{6} \mathrm{~A}$ RNA methylation modulators, which relates to the field of epigenetics, is also closely related to HCC prognosis. Based on the expression of $\mathrm{m}^{6} \mathrm{~A}$ RNA methylation modulators, two subgroups of HCC tissues were identified by consensus clustering. These clusters had different prognoses and clinicopathological characteristics. In addition, we used four selected $\mathrm{m}^{6} \mathrm{~A}$ RNA methylation modulators to derive a prognostic risk score, which was used to classify the HCC patients into high- and low-risk groups based on the median risk score.

Several studies have pointed out that the occurrence of liver cancer is related to the abnormal expression of $\mathrm{m}^{6} \mathrm{~A}$ RNA methylation modulators [20, 25, 27-29, 3537]. Zhao et al. [36] reported that increased YTHDF1 is related to poor prognosis of liver cancer patients, and YTHDF1 plays an important role in regulating liver cancer cell metabolism and cell cycle progression. Cheng and colleagues [27] reported that KIAA1429 facilitated HCC migration and invasion by inhibiting ID2 via

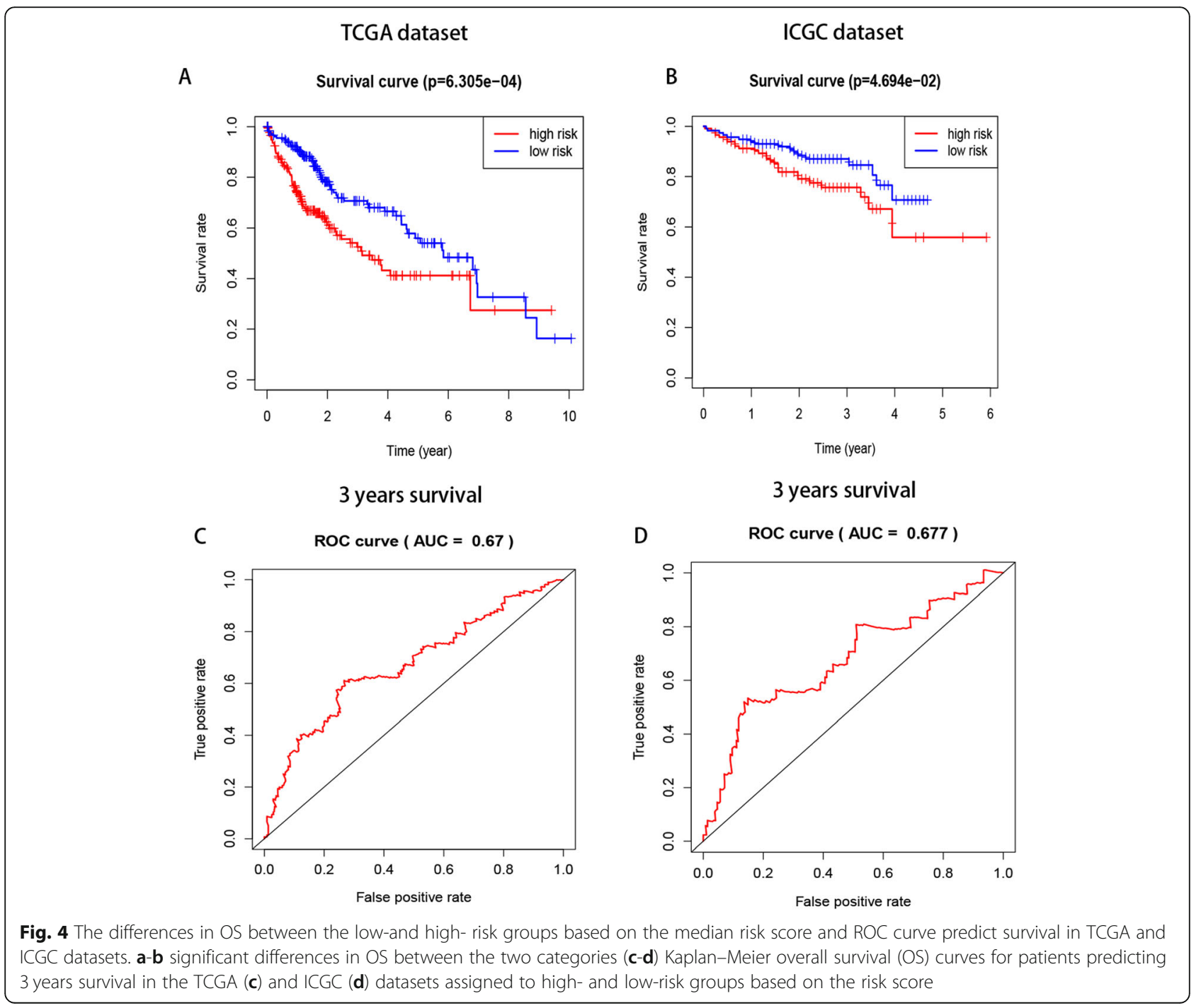




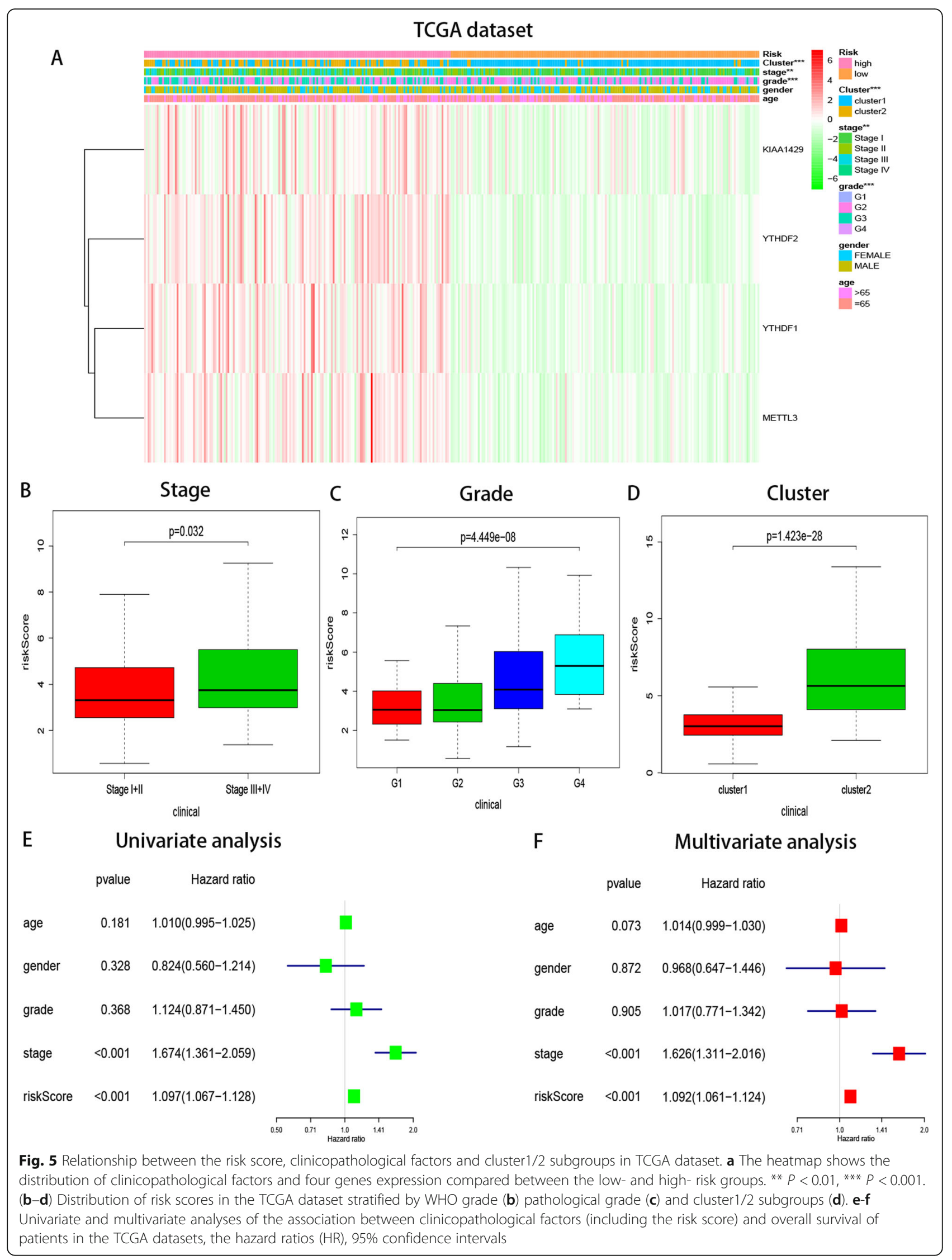




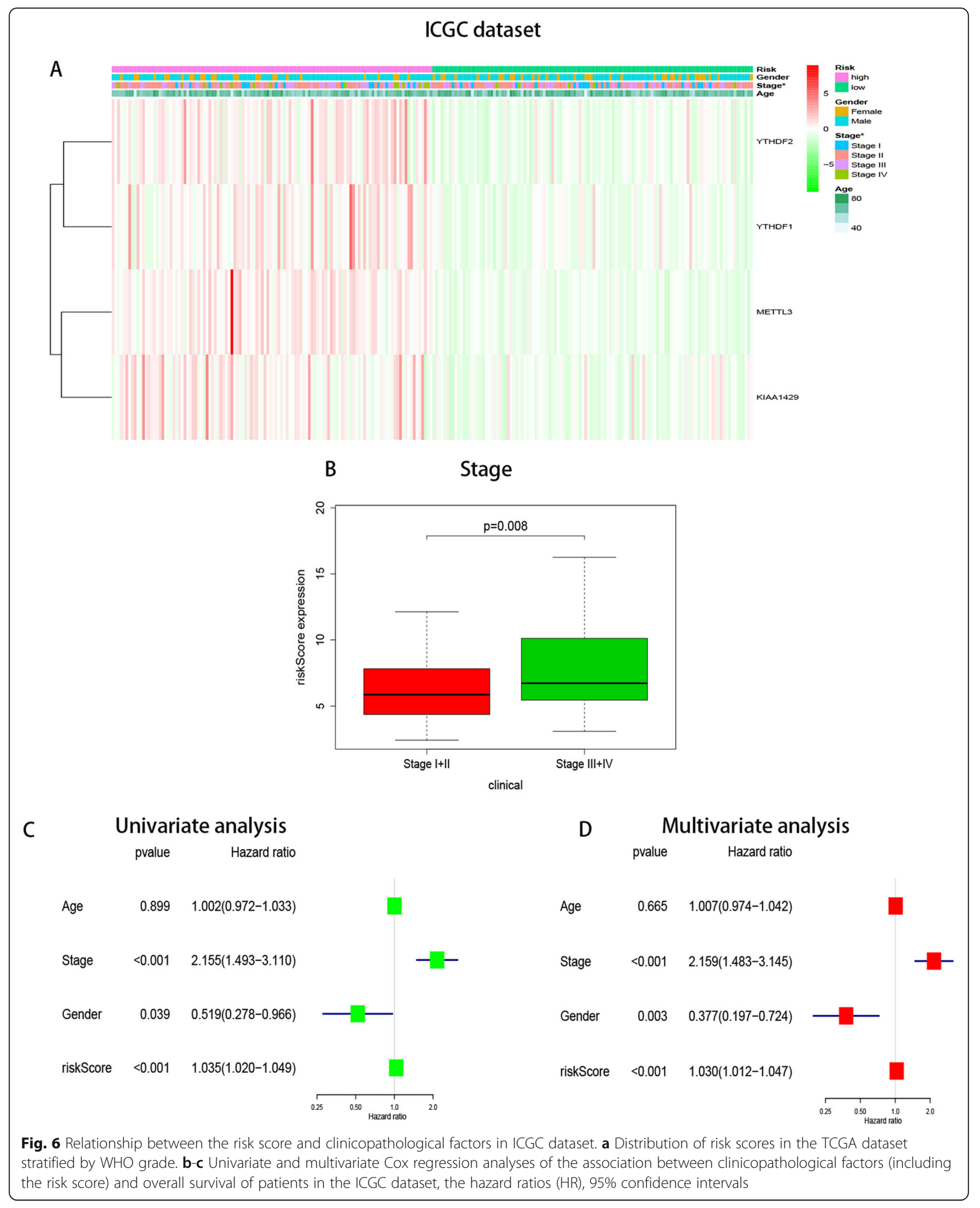


increasing $\mathrm{m}^{6} \mathrm{~A}$ modification of ID2 mRNA. Furthermore, Tanabe et al. [37] reported that YTHDC2 plays an important role in the growth of liver cancer cells.

The functions of METTL14, METTL3, and YTHDF2 in HCC are controversial. Ma et al. [20] demonstrated that METTL14 positively regulates the primary miRNA 126 (miR126) in an $\mathrm{m}^{6} \mathrm{~A}$-dependent manner by interacting with microprocessor complex subunit DiGeorge syndrome critical region 8 (DGCR8), and Ma et al. concluded that METTL14 can inhibit liver cancer metastasis. Ma et al. [20] also reported that METTL14 and $\mathrm{m}^{6} \mathrm{~A}$ levels were decreased in HCC tissues compared with normal tissues or tissues adjacent to HCC tissues, while METTL3 and WTAP levels were basically unchanged, In contrast, Chen et al. [29] reported that METTL14 levels were slightly higher in liver cancer tissues than in normal tissues, and METTL3 levels were considerably higher. Based on this, Chen and colleagues concluded that both METTL14 and METTL3 play carcinogenic roles in HCC, and they are necessary for HCC growth and metastasis. Zhong et al. [25] reported that YTHDF2 may play an anti-tumor role in HCC because its overexpression inhibited cell proliferation and growth and promoted the apoptosis of HCC cells. In contrast, Yang et al. [28] and Chen et al. [29] found that YTHDF2 played a pro-cancer role in HCC. These studies indicate that abnormal expression of $\mathrm{m}^{6} \mathrm{~A}$ RNA methylation modulators is closely related to HCC occurrence and development.

In this study, we comprehensively analyzed the expression of the 13 most common $\mathrm{m}^{6} \mathrm{~A}$ RNA methylation modulators in HCC tissues. There was no difference in the expression of METTL14 between HCC and normal tissues from either database, which is consistent with the results reported by Zhou et al. [35]. Additionally, we analyzed the CNV data of these 13 genes using the TCGA database. The HCC tissues were significantly different from the normal tissues in terms of $\mathrm{CNV}$. The expression of all 13 genes except HNRNPC was related to CNV. Only three genes (YTHDF1, YTHDC2, and KIAA1429) mainly exhibited increased copy numbers in the HCC tissues compared to the normal tissues, while the other 10 genes mainly exhibited reduced copy numbers. Furthermore, we analyzed data on SNP mutations in the 13 genes in HCC tissues and found very low mutation rates. These data indicate that abnormal expression of $\mathrm{m}^{6} \mathrm{~A}$ RNA methylation modulators in HCC tissues is not entirely caused by genetic changes $(\mathrm{CNV}$ or SNP mutations) [38].

Whether the expression of $\mathrm{m}^{6} \mathrm{~A}$ RNA methylation modulators can be used to predict cancer prognosis is an important research subject [7]. In this study, we used a risk signature constructed with four $\mathrm{m}^{6} \mathrm{~A}$ RNA methylation modulators (YTHDF1, YTHDF2, KIAA1429, and METTL3) to predict OS among HCC patients. In the TCGA database, patients with high risk scores were more likely to have a higher WHO stage and higher pathological grade and be in HCC subtype cluster 2. Also, in the ICGC database, high risk scores were associated with higher WHO stage. It should be noted that the risk score was independently associated with OS among HCC patients in both the TCGA and ICGC analyses. However, unlike in the TCGA analysis, sex was also an independent prognostic factor for $\mathrm{HCC}$ in the ICGC analysis, which may have been due to ethnic differences between the two datasets.

The METTL3 RNA methyltransferase is a "writer" protein responsible for $\mathrm{m}^{6} \mathrm{~A}$ modification and is involved in mRNA biogenesis, decay, and translation. METTL3 may play a carcinogenic role in lung cancer [39], bladder cancer $[23,40]$, gastric cancer [41], osteosarcoma [42], cutaneous squamous cell carcinoma [43], and acute myeloid leukemia (AML) [44]. Li et al. [45] reported that METTL3 promoted colorectal cancer progression through an $\mathrm{m}^{6} \mathrm{~A}$-IGF2BP2dependent mechanism, while Deng et al. [46] reported that METTL3 inhibited the proliferation and migration of colorectal cancer cells through the p38/ERK pathway. Additionally, Cui and colleagues [47] reported that METTL3 downregulation significantly promoted the growth, selfrenewal, and tumorigenesis of human glioblastoma stem cells, while METTL3 overexpression inhibited the growth and self-renewal of these cells. However, Visvanathan et al. [48] reported that METTL3 transcription was increased in glioblastoma, while METTL3 silencing inhibited tumor growth and prolonged the survival of mice. These results suggest that METTL3 may play varied roles in different types of cancer, and the study of METTL3 in colorectal cancer and glioblastoma remains controversial.

As a component of the $\mathrm{m}^{6} \mathrm{~A}$ "writer" complex, KIAA1429 is reported to be an RNA-binding protein involved in $\mathrm{m}^{6} \mathrm{~A}$ modification and RNA splicing and processing. At present, its role as an $\mathrm{m}^{6} \mathrm{~A}$ "writer" in tumorigenesis and its mechanism have not been fully reported. However, Cheng et al. [27] reported that KIAA1429 inhibits ID2 by increasing the $\mathrm{m}^{6} \mathrm{~A}$ modification of ID2 mRNA, thus promoting HCC migration and invasion. Additionally, Qian and colleagues [24] reported that KIAA1429 can regulate CDK1 in breast cancer in an $\mathrm{m}^{6} \mathrm{~A}$-independent manner, and act as a carcinogenic factor. These studies suggest that KIAA1429 promotes tumorigenesis and development.

YTH $\mathrm{N}^{6}$-methyladenosine RNA binding protein 1 (YTHDF1) is a member of the YTH domain family, which includes YTHDF1, 2, and 3 and YTHDC1 and 2. As a "reader" of $\mathrm{m}^{6} \mathrm{~A}$-modified mRNA, cytoplasmic YTHDF1 interacts with binding sites on $\mathrm{m}^{6} \mathrm{~A}$-modified mRNA to promote the initiation of translation [49]. However, the link between YTHDF1 and cancer is largely unknown. Han et al. [50] reported that $\mathrm{m}^{6} \mathrm{~A}$ RNA modification, involving YTHDF1, modulates the anti-tumor immune response. Zhao et al. [36] and Zhou et al. [35] reported that YTHDF1 is highly expressed in liver cancer and is significantly 
associated with poor prognosis. Furthermore, Nishizawa et al. [51] and Bai et al. [22] reported that YTHDF1 is highly expressed in colorectal cancer and plays an important role in carcinogenesis.

The main role of YTHDF2 is to regulate the degradation of $\mathrm{m}^{6} \mathrm{~A}$-modified mRNAs [24]. However, the relationship between YTHDF2 and cancer is largely unknown. Yang et al. [28] reported that miR-145 regulates $\mathrm{m}^{6} \mathrm{~A}$ by targeting the 3 '-untranslated region of YTHDF2 in HCC cells, and YTHDF2 expression is closely related to the malignant degree of HCC. Thereafter, Chen and colleagues [52] reported that YTHDF2 was highly expressed in pancreatic cancer, which promoted the proliferation and inhibited the migration and invasion of pancreatic cancer cells. Furthermore, many studies have focused on the relationship between YTHDF2 and AML, indicating that YTHDF2 is increased in the broad spectrum of human AML tissues. Targeting YTHDF2 to inhibit its expression can enlarge hematopoietic stem cells, enhance bone marrow reconstruction, and selectively impair AML [53-55], suggesting that it may be useful in the treatment of hematological malignant tumors.

\section{Conclusions}

Our results systematically demonstrate the expression of $13 \mathrm{~m}^{6} \mathrm{~A}$ RNA methylation modulators in HCC, reveal the CNV and SNP mutations of these genes in HCC, and clarify the prognostic value of the $\mathrm{m}^{6} \mathrm{~A}$ RNA methylation modulators. Our study provides important information for further exploration of the role of $\mathrm{m}^{6} \mathrm{~A}$ RNA methylation in HCC.

\section{Supplementary information}

Supplementary information accompanies this paper at https://doi.org/10. 1186/s12885-020-6638-5.

Additional file 1: Figure S1. CNV copy number variation and SNP mutations of m6A RNA methylation modulators in HCC. (A) CNV copy number variation of the 13 genes in HCC tissues. Dioploid means normal; Shallow deletion means deletion of one copy number; Copy number gain means get a copy number; Amplification means get two or more copies. (B) SNP mutations of the 13 genes in HCC tissues. The vertical coordinate represents each gene, and the horizontal coordinate represents the number and type of SNP mutations of each gene in 364 tumor samples.

Additional file 2: Figure S2. The relationship between mRNA expression of the 13 genes and copy number. (A-M) Kruskal-Wallis test was used for the differences among the groups, Single deletion means deletion of one copy number; Single gain means get a copy number; Amplification means get two or more copies.The numbers in the figures represent the sample size for each category of CNV

Additional file 3: FigureS3. Consensus clustering of m6A RNA methylation modulators identified two clusters of hepatocellular carcinoma and gene differential expression between two clusters, (A)Consensus clustering cumulative distribution function (CDF) for $\mathrm{k}=2$ to 9. (B) Relative change in area under CDF curve for $k=2$ to 9. (C-D) Consensus clustering matrix for $\mathrm{k}=2(\mathrm{C})$ and $\mathrm{k}=3(\mathrm{D})$. (E) The tracking plot for $k=2$ to $k=9$. (F) Heatmap of expression of m6A RNA methylation modulators of the two clusters, gene differential expression was tested by Wilcox test, ${ }^{*} P<0.05$ and ${ }^{* *} P<0.001$.

Additional file 4: Figure S4. Risk signature with four common m6A RNA methylation modulators. (A-B) The process of building the signature containing four m6A RNA methylation modulators in two datasets. The hazard ratios (HR), 95\% confidence intervals (Cl) calculated by univariate Cox regression and the coefficients calculated by multivariate Cox regression using LASSO are shown.

Additional file 5: Figure S5. Lasso risk regression model risk diagram in TCGA dataset. (A) Heatmap of the expression levels of the four selected m6A RNA methylation modulators in high- and low-risk patients. (B) Survival status map, the green dots on behalf of survival state, the red dots on behalf of death state. (C)Nomograms based on the risk score and clinicopathological factors to predict 1-, 3-, and 5-year survival rates.

Additional file 6: Figure S6. Lasso risk regression model risk diagram in ICGC dataset. (A) Heatmap of the expression levels of the four selected m6A RNA methylation modulators in high- and low-risk patients. (B) Survival status map, the green dots on behalf of survival state, the red dots on behalf of death state. (C)Nomograms based on the risk score and clinicopathological factors to predict 1-, 3-, and 5-year survival rates.

Additional file 7: Figure S7. Nomograms combining risk values with clinicopathological factors to predict survival. (A) A comprehensive nomogram composed of age and stage combined with risk values, was used to predict 1-year, 3-year and 5-year survival rates in the TCGA database. (B)A comprehensive nomogram composed of age, stage and gender combined with risk values was used to predict the 1-year, 3-year and 5-year survival rate In the ICGC database.

Additional file 8: Table S1. Summary of expression and genetic changes of m6A RNA methylation modulators in HCC.

Additional file 9: Table S2. Clinicopathological features of patients included in this study.

\section{Abbreviations}

AML: Acute myeloid leukemia; CNV: Copy number variation; HBV: Hepatitis B virus; HCC: Hepatocellular carcinoma; HCV: Hepatitis C virus; HR: Hazard ratio; HTSeq: High-throughput sequencing; ICGC: International Cancer Genome Consortium; LASSO: Least absolute shrinkage and selection operator; miRNA: microRNA; mRNA: Messenger RNA; OS: Overall survival; PCA: Principal component analysis; ROC: Receiver operating characteristic; SNP: Singlenucleotide polymorphism; TCGA: The Cancer Genome Atlas; WHO: World Health Organization; YTHDF: YTH N ${ }^{6}$-methyladenosine RNA binding protein

\section{Acknowledgements}

The authors gratefully acknowledge contributions from the TCGA and ICGC networks.

\section{Authors' contributions}

QNF was responsible for conceptualizing and designing the study, data collection and analysis, and preparing the graphs, and was a major contributor to writing the manuscript; WGQ interpreted the data; TC was responsible for the statistical analysis; LZC was responsible for the bioinformatics analysis; BXT processed the charts and tables in the revision process of the later articles; QSY and ZXM contributed to the critical review of the manuscript; JHX supervised and contributed to the critical review of the manuscript. All authors read and approved the final manuscript.

\section{Funding}

The present study was supported by grants from National Natural Science Foundation of China (819601 19, 81660107 and 81802478), and Guangxi Natural Science Foundation(2018GXNSFAA281221 and 2018GXNSFBA050042). The funding bodies were not involved in the design of this study, in the collection, analysis, and interpretation of the data, or in writing of the manuscript.

Availability of data and materials

All analyzed data are included in this published article and its supplementary information file. The original data are available upon request to the corresponding author. 


\section{Ethics approval and consent to participate}

Not applicable. All data in this study are publicly available.

\section{Consent for publication}

Not applicable.

\section{Competing interests}

The authors declare that they have no competing interests.

\section{Author details}

'Department of Gastroenterology, The First Affiliated Hospital of Guangxi Medical University, Nanning 530021, China. ${ }^{2}$ Department of Gastroenterology, Affiliated Hospital of Guilin Medical University, Guilin 541000, China. ${ }^{3}$ Department of Pathology, Affiliated Hospital of Guilin Medical University, Guilin 541000, China. ${ }^{4}$ Department of Radiotherapy, Affiliated Hospital of Guilin Medical University, Guilin 541000, China. ${ }^{5}$ School of Public Health, Guilin Medical University, Guilin 541000, China. ${ }^{6}$ Department of B Ultrasound, Affiliated Hospital of Guilin Medical University, Guilin 541000, China.

Received: 30 October 2019 Accepted: 17 February 2020

Published online: 28 February 2020

\section{References}

1. Chen W, Zheng R, Baade PD, Zhang S, Zeng H, Bray F, Jemal A, Yu XQ, He J. Cancer statistics in China, 2015. CA Cancer J Clin. 2016;66(2):115-32.

2. Torre LA, Bray F, Siegel RL, Ferlay J, Lortet-Tieulent J, Jemal A. Global cancer statistics, 2012. CA Cancer J Clin. 2015;65(2):87-108.

3. Thorgeirsson SS, Grisham JW. Molecular pathogenesis of human hepatocellular carcinoma. Nat Genet. 2002;31(4):339-46.

4. Shen J, He L, Li C, Wen T, Chen W, Lu C, Yan L, Li B, Yang J. Nomograms to predict the individual survival of patients with solitary hepatocellular carcinoma after hepatectomy. Gut Liver. 2017;11(5):684-92.

5. Lee J, Lee $\mathrm{JH}$, Yoon $\mathrm{H}$, Lee $\mathrm{HJ}$, Jeon $\mathrm{H}$, Nam J. Extraordinary radiation supersensitivity accompanying with sorafenib combination therapy: what lies beneath? Radiat Oncol J. 2017:35(2):185-8.

6. Hsiao JH, Tsai CC, Liang TJ, Chiang CL, Liang HL, Chen IS, Chen YC, Chang PM, Chou NH, Wang BW. Adjuvant hepatic arterial infusion chemotherapy is beneficial for selective patients with hepatocellular carcinoma undergoing surgical treatment. Int J Surg. 2017:45:35-41.

7. Pan Y, Ma P, Liu Y, Li W, Shu Y. Multiple functions of m(6)A RNA methylation in cancer. J Hematol Oncol. 2018;11(1):48.

8. Desrosiers R, Friderici K, Rottman F. Identification of methylated nucleosides in messenger RNA from Novikoff hepatoma cells. Proc Natl Acad Sci U S A. 1974;71(10):3971-5.

9. Alarcon CR, Lee H, Goodarzi H, Halberg N, Tavazoie SF. N6-methyladenosine marks primary microRNAs for processing. Nature. 2015:519(7544):482-5.

10. Patil DP, Chen CK, Pickering BF, Chow A, Jackson C, Guttman M, Jaffrey SR. m(6)A RNA methylation promotes XIST-mediated transcriptional repression. Nature. 2016;537(7620):369-73.

11. Kane SE, Beemon K. Precise localization of m6A in Rous sarcoma virus RNA reveals clustering of methylation sites: implications for RNA processing. Mol Cell Biol. 1985;5(9):2298-306.

12. Dominissini D, Moshitch-Moshkovitz S, Schwartz S, Salmon-Divon M, Ungar L, Osenberg S, Cesarkas K, Jacob-Hirsch J, Amariglio N, Kupiec M, et al. Topology of the human and mouse m6A RNA methylomes revealed by m6A-seq. Nature. 2012;485(7397):201-6.

13. Roignant JY, Soller M. m(6)A in mRNA: an ancient mechanism for finetuning gene expression. Trends Genet. 2017;33(6):380-90.

14. Yue Y, Liu J, He C. RNA N6-methyladenosine methylation in posttranscriptional gene expression regulation. Genes Dev. 2015;29(13):1343-55.

15. Lan Q, Liu PY, Haase J. The critical role of RNA m(6)A methylation in Cancer. Andrology. 2019;79(7):1285-92

16. Fitzsimmons CM, Batista PJ. It's complicated... m(6)A-dependent regulation of gene expression in cancer. Oncogene. 2019;1862(3):382-93.

17. Arguello AE, DeLiberto AN, Kleiner RE. RNA chemical proteomics reveals the $\mathrm{N}(6)$-Methyladenosine (m(6)a)-regulated protein-RNA Interactome. J Am Chem Soc. 2017;139(48):17249-52.

18. Wang $S$, Chai $P$, Jia R, Jia R. Novel insights on m(6)A RNA methylation in tumorigenesis: a double-edged sword. Nat Cell Biol. 2018;17(1):101.
19. Zhao BS, Roundtree IA, He C. Post-transcriptional gene regulation by mRNA modifications. Nat Rev Mol Cell Biol. 2017;18(1):31-42.

20. Ma JZ, Yang F, Zhou CC, Liu F, Yuan JH, Wang F, Wang TT, Xu QG, Zhou WP, Sun SH. METTL14 suppresses the metastatic potential of hepatocellular carcinoma by modulating N(6) -methyladenosine-dependent primary MicroRNA processing. Hepatology (Baltimore, Md). 2017;65(2):529-43.

21. Huang G, Shen B, Ju Z, Li Z, Qian P, Shao W, Shi H, He XC, Gogol M, Yu Z, et al. Author correction: suppression of $m(6) A$ reader $Y$ thdf2 promotes hematopoietic stem cell expansion. Cell Res. 2018;28(10):1042.

22. Bai Y, Yang C, Wu R, Huang L, Song S, Li W, Yan P, Lin C, Li D, Zhang Y. YTHDF1 regulates Tumorigenicity and Cancer stem cell-like activity in human colorectal carcinoma. Mol Cancer. 2019;9:332.

23. Cheng $M$, Sheng $L$, Gao Q, Xiong Q, Zhang $H$, Wu M, Liang $Y$, Zhu F, Zhang $Y$, Zhang $X$, et al. The $\mathrm{m}(6) A$ methyltransferase METTL3 promotes bladder cancer progression via AFF4/NF-kappaB/MYC signaling network. Oncogene. 2019;38(19):3667-80

24. Qian JY, Gao J, Sun X, Cao MD, Shi L, Xia TS, Zhou WB, Wang S, Ding Q, Wei JF. KIAA1429 acts as an oncogenic factor in breast cancer by regulating CDK1 in an N6-methyladenosine-independent manner. Oncogene. 2019; 38(33):6123-41.

25. Zhong L, Liao D, Zhang M, Zeng C, Li X, Zhang R, Ma H, Kang T. YTHDF2 suppresses cell proliferation and growth via destabilizing the EGFR mRNA in hepatocellular carcinoma. Nat Commun. 2019;442:252-61.

26. Rong ZX, Li Z, He JJ, Liu LY, Ren XX, Gao J, Mu Y, Guan YD, Duan YM, Zhang $X P$, et al. Downregulation of fat mass and obesity associated (FTO) promotes the progression of intrahepatic Cholangiocarcinoma. Front Oncol. 2019;9:369.

27. Cheng X, Li M, Rao X, Zhang W, Li X, Wang L, Huang G. KIAA1429 regulates the migration and invasion of hepatocellular carcinoma by altering $\mathrm{m} 6 \mathrm{~A}$ modification of ID2 mRNA. OncoTargets and therapy. 2019;12:3421-8.

28. Yang Z, Li J, Feng G, Gao S, Wang Y, Zhang S, Liu Y, Ye L, Li Y, Zhang X. MicroRNA-145 Modulates N(6)-Methyladenosine Levels by Targeting the 3'Untranslated mRNA Region of the N(6)-Methyladenosine Binding YTH Domain Family 2 Protein. Hepatology (Baltimore, Md). 2017;292(9):3614-23.

29. Chen M, Wei L, Law CT, Tsang FH, Shen J, Cheng CL, Tsang LH, Ho DW Chiu DK, Lee JM, et al. RNA N6-Methyladenosine methyltransferase-like 3 promotes liver Cancer progression through YTHDF2Dependent posttranscriptional silencing of SOCS2. Hepatology. 2018;67(6):2254-70.

30. Wang L, Shi J, Huang Y, Liu S, Zhang J, Ding H, Yang J, Chen Z. A six-gene prognostic model predicts overall survival in bladder cancer patients. Cancer Cell Int. 2019;19:229.

31. Chai R-C, Wu F, Wang QX, Zhang S, Zhang KN, Liu YQ, Zhao Z, Jiang T, Wang YZ, Kang CS, et al. m6A RNA methylation regulators contribute to malignant progression and have clinical prognostic impact in gliomas. Aging (Albany NY). 2019;11(4):1204-25.

32. Schulze K, Imbeaud S, Letouze E, Alexandrov LB, Calderaro J, Rebouissou S, Couchy G, Meiller C, Shinde J, Soysouvanh F, et al. Exome sequencing of hepatocellular carcinomas identifies new mutational signatures and potential therapeutic targets. Nat Genet. 2015;47(5):505-11.

33. Boccaletto P, Machnicka MA, Purta E, Piatkowski P, Baginski B, Wirecki TK, de Crecy-Lagard V, Ross R, Limbach PA, Kotter A, et al. MODOMICS: a database of RNA modification pathways. 2017 update. Nucleic Acids Res. 2018;46(D1): D303-7.

34. Deng $X$, Su R, Feng X, Wei $M$, Chen J. Role of N(6)-methyladenosine modification in cancer. Curr Opin Genet Dev. 2018;48:1-7.

35. Zhou Y, Yin Z, Hou B, Yu M, Chen R, Jin H, Jian Z. Expression profiles and prognostic significance of RNA N6-methyladenosine-related genes in patients with hepatocellular carcinoma: evidence from independent datasets. Cancer Manag Res. 2019;11:3921-31.

36. Zhao X, Chen $Y$, Mao Q, Jiang X, Jiang W, Chen J, Xu W, Zhong L, Sun X. Overexpression of YTHDF1 is associated with poor prognosis in patients with hepatocellular carcinoma. Cancer Biomark. 2018;21(4):859-68.

37. Tanabe A, Konno J, Tanikawa K, Sahara H. Transcriptional machinery of TNFalpha-inducible YTH domain containing 2 (YTHDC2) gene. Gene. 2014; 535(1):24-32

38. Jia P, Zhao Z. Impacts of somatic mutations on gene expression: an association perspective. Brief Bioinform. 2017;18(3):413-25.

39. Lin S, Choe J, Du P, Triboulet R, Gregory RI. The m(6)A methyltransferase METTL3 promotes translation in human Cancer cells. Mol Cell. 2016;62(3): 335-45.

40. Han J, Wang JZ, Yang X, Yu H, Zhou R, Lu HC, Yuan WB, Lu JC, Zhou ZJ, Lu $\mathrm{Q}$, et al. METTL3 promote tumor proliferation of bladder cancer by 
accelerating pri-miR221/222 maturation in m6A-dependent manner. Mol Cancer. 2019;18(1):110.

41. Liu T, Yang S. Dysregulated N6-methyladenosine methylation writer METTL3 contributes to the proliferation and migration of gastric cancer. J Cell Physio. 2020;235(1):548-62.

42. Miao W, Chen J, Jia L, Ma J, Song D. The m6A methyltransferase METTL3 promotes osteosarcoma progression by regulating the m6A level of LEF1. Biochem Biophys Res Commun. 2019;516(3):719-25.

43. Zhou R, Gao Y, Lv D, Wang C, Wang D, Li Q. METTL3 mediated m(6)A modification plays an oncogenic role in cutaneous squamous cell carcinoma by regulating DeltaNp63. Mol Cancer. 2019;515(2):310-7.

44. Barbieri I, Tzelepis K, Pandolfini L, Shi J, Millan-Zambrano G, Robson SC, Aspris D, Migliori V, Bannister AJ, Han N, et al. Promoter-bound METTL3 maintains myeloid leukaemia by m(6)A-dependent translation control. Nature. 2017:552(7683):126-31

45. Li T, Hu PS, Zuo Z, Lin JF, Li X, Wu QN, Chen ZH, Zeng ZL, Wang F, Zheng J, et al. METTL3 facilitates tumor progression via an m(6)A-IGF2BP2-dependent mechanism in colorectal carcinoma. Nat Commun. 2019;18(1):112.

46. Deng R, Cheng Y, Ye S, Zhang J, Huang R, Li P, Liu H, Deng Q, Wu X, Lan P, et al. m(6)A methyltransferase METTL3 suppresses colorectal cancer proliferation and migration through p38/ERK pathways. OncoTargets and therapy. 2019;12:4391-402.

47. Cui Q, Shi H, Ye P, Li L, Qu Q, Sun G, Sun G, Lu Z, Huang Y, Yang CG, et al. $m(6) A$ RNA methylation regulates the self-renewal and tumorigenesis of Glioblastoma stem cells. Cell Rep. 2017;18(11):2622-34.

48. Visvanathan A, Patil V, Arora A, Hegde AS, Arivazhagan A, Santosh V, Somasundaram K. Essential role of METTL3-mediated $m(6) A$ modification in glioma stem-like cells maintenance and radioresistance. Oncogene. 2018; 37(4):522-33.

49. Wang X, Zhao BS, Roundtree IA, Lu Z, Han D, Ma H, Weng X, Chen K, Shi H, He C. N(6)-methyladenosine modulates messenger RNA translation efficiency. Cell. 2015;161(6):1388-99.

50. Han D, Liu J, Chen C, Dong L, Liu Y, Chang R, Huang X, Liu Y, Wang J, Dougherty $\mathrm{U}$, et al. Author correction: anti-tumour immunity controlled through mRNA m(6)A methylation and YTHDF1 in dendritic cells. Biochemistry. 2019;568(7751):E3.

51. Nishizawa Y, Konno M, Asai A, Koseki J, Kawamoto K, Miyoshi N, Takahashi H, Nishida N, Haraguchi N, Sakai D, et al. Oncogene c-Myc promotes epitranscriptome m(6)A reader YTHDF1 expression in colorectal cancer. Oncotarget. 2018;9(7):7476-86.

52. Chen J, Sun Y, Xu X, Wang D, He J, Zhou H, Lu Y, Zeng J, Du F, Gong A, et al. YTH domain family 2 orchestrates epithelial-mesenchymal transition/ proliferation dichotomy in pancreatic cancer cells. Cell Cycle. 2017;16(23): 2259-71.

53. Paris J, Morgan M, Campos J, Spencer GJ, Shmakova A, Ivanova I, Mapperley C, Lawson H, Wotherspoon DA, Sepulveda C, et al. Targeting the RNA m(6)A Reader YTHDF2 Selectively Compromises Cancer Stem Cells in Acute Myeloid Leukemia. Cell stem cell. 2019;25(1):137-48 e136.

54. Wang H, Zuo H, Liu J, Wen F. Loss of YTHDF2-mediated m(6)A-dependent mRNA clearance facilitates hematopoietic stem cell regeneration. Cell Res. 2018;28(10):1035-8.

55. Li J, Meng S, Xu M, Wang S, He L, Xu X, Wang X, Xie L. Downregulation of $N(6)$-methyladenosine binding YTHDF2 protein mediated by miR-493-3p suppresses prostate cancer by elevating N(6)-methyladenosine levels. Cell Res. 2018;9(3):3752-64.

\section{Publisher's Note}

Springer Nature remains neutral with regard to jurisdictional claims in published maps and institutional affiliations.

Ready to submit your research? Choose BMC and benefit from:
- fast, convenient online submission
- thorough peer review by experienced researchers in your field
- rapid publication on acceptance
- support for research data, including large and complex data types
- gold Open Access which fosters wider collaboration and increased citations
- maximum visibility for your research: over 100M website views per year
At BMC, research is always in progress.
Learn more biomedcentral.com/submissions

